\title{
Single implant supported crown opposing a supraerupted tooth
}

\author{
Authors \\ Tanuj Minocha ${ }^{1 *}$, Noopur Rathi ${ }^{2}$, Shailesh Jain ${ }^{3}$ \\ ${ }^{1}$ Assistant Professor, Department of Periodontics, DJ College of Dental Sciences, BRAU \\ ${ }^{2}$ Assistant Professor, Department of Prosthodontics, DJ College of Dental Sciences, BRAU \\ ${ }^{3}$ Assistant Professor, Department of Prosthodontics, Sharda School of Dental Sciences, SU \\ *Corresponding Author \\ Tanuj Minocha
}

\begin{abstract}
From conventional fixed partial denture to conventional cast partial dentures, the availability of a single tooth implant restoration has brought a new set of conservative standards in prosthetic dentistry. The use of osseointegrated single tooth implants has become as the first treatment option that every clinician prefers to advise to his patient. We present a case of a missing right mandibular first molar which was successfully restored with a single tooth implant using a cemented abutment with a difference that its opposing tooth was supraerupted beyond occlusal plane. The implant was placed in a two stage surgical procedure and a porcelain fused to metal with full porcelain coverage was provided on the crown.

Keywords: supraeruption, abutment, screw/ cement retained, osseointegration.
\end{abstract}

\section{Introduction}

The inspirational work by Branemark et al in 1965, ${ }^{1}$ introduced the concept of osseointegration between a viable bone and biocompatible alloy in dentistry. In the future decades to come, dentistry witnessed large scale research that led to the evolution of various prosthodontic options for replacing natural teeth. The science of fixed partial denture that required to prepare a natural tooth structure began to look a radical treatment for a minimum of two healthy natural teeth were sacrificed to retain a prosthesis. This was mainly due to application of osseointegration in replacing a single missing tooth with a single tooth implant treatment procedure. However, there has been some controversy and debate for single tooth implants also., ${ }^{2,3}$ Various issues related to single tooth implants revolved around marginal bone loss, bone quantity and quality, absence of keratinized tissue, implant pocket formation and the patients' plaque control habits. ${ }^{4-7}$ Application of single tooth implants in the anterior maxillary region received a lot of attention to the soft tissue aesthetics which gave rise to the concept of emergence profile of implants. Various abutment designs evolved as a result of those concepts. $^{8}$ The role of occlusion has been considered as a single determining factor for long term success of implant supported prosthesis. Implants are to be indicated more or less in an ideal occlusion. ${ }^{9}$ However, there are some situations where the occlusal discrepancy is not ideal but can be corrected during the implant therapy.

Through this case report we intend to present a case of a single tooth implant that replaced a lost right first mandibular molar, with opposing molar on the same side being supraerupted and intruding the occlusal plane by several millimeters on the buccal and the palatal side. 


\section{Case Report}

An adult male patient aged 44 years reported to the department of periodontics for completion of his previously planned dental treatment with implant supported single crown restorations in relation to the mandibular arch. The patient had already received a single implant supported crown 6 months back and since he was extremely satisfied with the outcome of his first implant experience, he had decided to complete the treatment with a same prosthetic option. No variation in medical, social and drug histories were reported by him between the time from his previous treatment. Extra oral examination revealed normal clinical features while intra oral examination disclosed a kennedy class 3 partial edentulous space on the right side of the mandible with mandibular right first molar missing. The mandibular left first molar was previously restored with a single implants supported prosthesis (Fig 1 A). The patient was referred to the department of prosthodontics where diagnostic impressions were made with irreversible hydrocolloid (Jeltrate Alginate, Fast Set; Dentsply Intl, York, Pa) and the poured casts (Pankaj Industries, Mumbai, India) were retrieved. A surgical guide was fabricated and the ideal position of the implant was verified on an orthopantomogram radiographic procedure using a ball bearing technique (Fig 1 B). Implant fixture of the CeraOne system (Nobel Bio care, Goteborg, Sweden) was placed in stage 1 surgery and after a period of four months implant fixtures were located and its alignment verified during stage 2 surgery (Fig 1 C, D). The angulation of an implant fixture was verified using a guide pin following which an abutment (cement retained) was placed on the implant fixture. The tissues were allowed to heal for a period of two weeks, after which the fabrication of cement crown restoration was initiated (Fig 2 A). Before making the final implant level impression using an elastomeric impression material

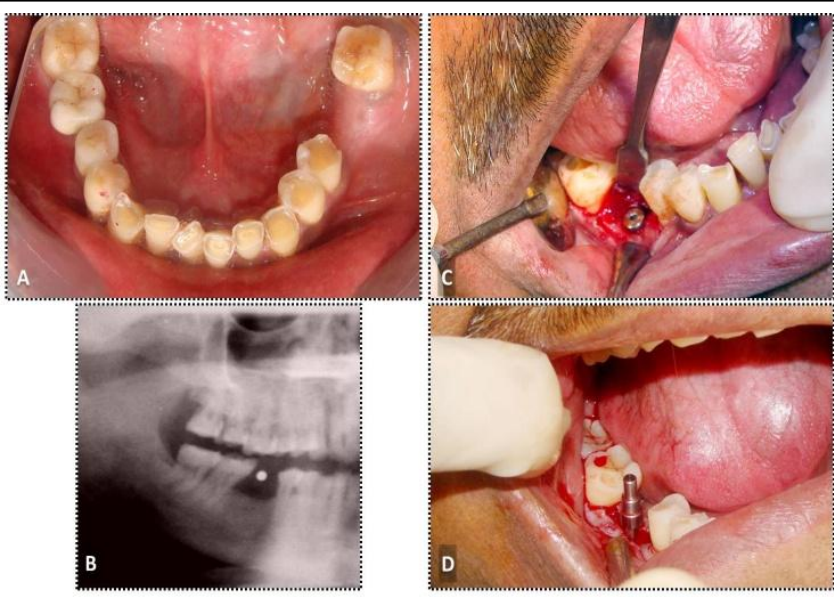

Figure 1: (A) Intra oral view of missing tooth (B) Radiograph with a surgical template (C) Implant fixture location and (D) Verification of implant alignment

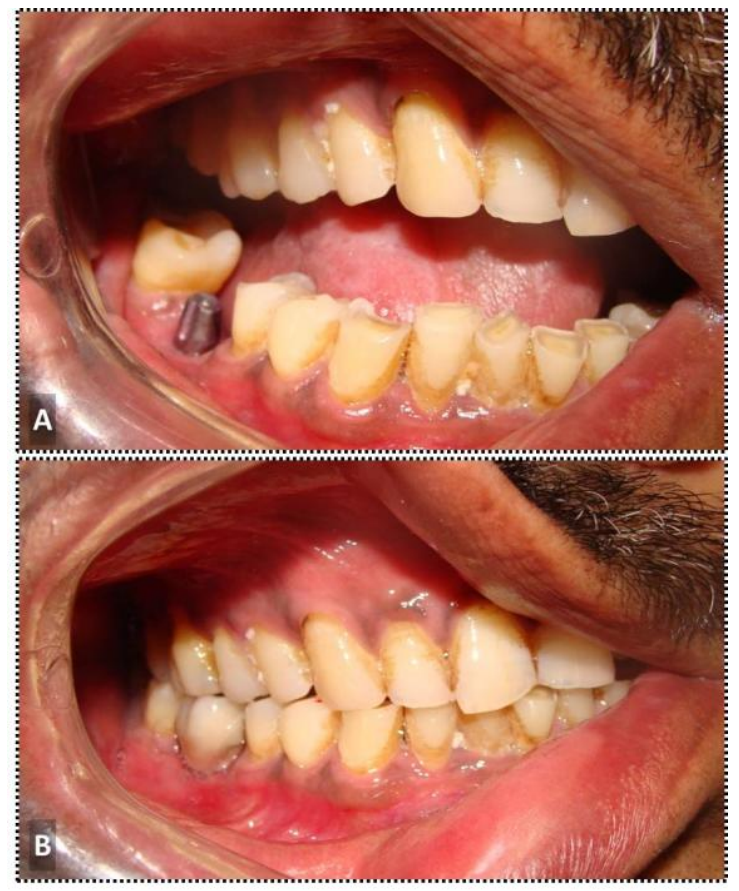

Figure 2: (A) Cemented abutment screwed to implant fixture (B) Porcelain fused to metal crown cemented onto the implant abutment

(Extrude and Extrude Extra; Kerr Corp), the opposing molar was corrected using the clinical occlusal equilibration procedure. Determination of correction was done after evaluating occlusion on a programmed semi adjustable articulator (Whip Mix series 3000; Elite Dental Services, Inc, Orlando, Fla). A single porcelain fused to metal crown with occlusal porcelain was fabricated in the laboratory and cemented onto the implant abutment (Fig 2 B) with zinc polycarboxylate cement (Poly F Plus; Dentsply DeTrey GmbH, Konstanz, Germany). The 
occlusion was evaluated in centric and eccentric movements. The patient was given oral hygiene maintenance instructions, and was put on a follow up. During his subsequent appointments the patient expressed his satisfaction with the outcome of his implant supported crown.

\section{Discussion}

The choice of a single tooth implant restoration seems to be unanimous if a patient has experienced it once. The case described in this article is one such example, since the patient was already using a single implant supported prosthesis. Patients desire is an important clinical consideration when implant supported crowns are to be given in natural dentition. Patients who desire for things are bound to perform its maintainence and care. This was evident in this case since earlier implant and the rest of natural dentition was maintained according to desired patient performance. For a practitioner, choosing the appropriate abutment either for the single implant supported crown or for removable prosthetic options is not the only confusing task, but a tricky one too. ${ }^{10,11}$ Poor performance of the abutment can result in prosthetic failure and practitioners may compromise abutment quality for more financial benefits or not making financially hard for the patient. While informing the patient about the cost of the restoration one should always keep in mind the choice of abutment that will be made. The ideal abutment is the one whose mechanical properties and stress distribution are well known by the practitioner. ${ }^{12}$

Another important aspect of this case report is the need of correcting the occlusal plane before fabrication of the prosthesis. The tooth opposing the edentulous space had supraerupted which resulted in the intrusion of freeway space as well. Conservative occlusal equilibration can be achieved only through analysis of the programmed semi adjustable or a fully adjustable articulator. We corrected the occlusion on the articulator following which we prepared an acrylic template which guided correct occlusal equilibration procedure in the patient.

\section{Conclusion}

Convincing a patient for a first time needs some effort, but very little effort is required for a patient who has already experienced an implant supported prosthesis. occlusal plane correction is mandatory before planning occlusion in implant supported prosthesis.

\section{Acknowledgements}

The authors would like to acknowledge the staff of the department of prosthodontics and the staff of the dental laboratory for their valued cooperation.

\section{References}

1. Brånemark PI, Adell R, Hansson $\mathrm{BO}$, et al. Intraosseous anchorage of dental prosthesis: I-experimental studies. Scand J Plast Reconstr Surg 1969;3:81-100.

2. Hutter JW. Implants versus the natural tooth: stand up for what we know is right. J Endod 2001;27:637.

3. Felton DA. Implant or root canal therapy: a prosthodontist's view. J Esthet Restor Dent 2005; 17:197-9.

4. Jaffin RA, Berman CL. The excessive loss of Brånemark fixtures in Type IV bone: A 5year analysis. J Periodontol 1991;62:2-4.

5. Block MS, Gardiner D, Kent JN, Misiek DJ, Finger IM, Guerra L. Hydroxyapatite-coated cylindrical implants in the posterior mandible: 10-year observations. Int J Oral Maxillofac Implants 1996;11:626-633.

6. Singh M, Mattoo KA, Jain S. Replacement of a mandibular molar with implant retained single crown. Oral Surgery Oral Medicine Oral Radiology 2014;2:25-27

7. Albrektsson T, Zarb GA, Worthington P, et al. The long term efficacy of currently used dental implants: a review and proposed criteria of success. Int $\mathbf{J}$ Oral Maxillofac Implants 1986;1:11.

8. Hebel KS, Gajjar RC. Cement-retained versus screw retained implant restorations: Achieving optimal occlusion and esthetics in 
implant dentistry. J Prosthet Dent 1997;77:

28-35.

9. Mattoo K, Garg R, Bansal V. Designing the occlusion for a single tooth implant in a compromised occlusion. Journal of Medical Sciences and Clinical Research 2014;2:2996-3000

10. Taylor TD, Agar JR, Vogiatzi T. Implant prosthodontics: Current perspectives and future directions. Int $\mathbf{J}$ Oral Maxillofac Implants 2000;15:66-75.

11. Mattoo K, Singh SP, Brar A. A simple technique to align attachment components in implant supported mandibular overdenture. International Journal Of Research In Medical Sciences And Technology 2014;1:6-8

12. Albrektsson T, Dahl E, Enbom L, et al. Osseointegrated oral implants. A Swedish multicenter study of 8139 consecutively inserted Nobelpharma implants. J Periodontol 1988;59:287-296. 